\title{
Persistent SCTP Timeout Policy by Using Cross-LAYer MECHANISM
}

\author{
Mahamadou ISSOUFOU TIADO ${ }^{1}$, Hamadou SALIAH-HASSANE ${ }^{2}$ \\ ${ }^{1}$ Computer Science Department, Université Abdou Moumouni, Niamey, Niger \\ ${ }^{2}$ Science and Technology Department \& LICEF Research Center \\ TELUQ | University of Quebec \\ 100 Sherbrooke Street West, Montreal, Quebec \\ $\mathrm{H} 2 \mathrm{X} 3 \mathrm{P} 2$, CANADA
}

\begin{abstract}
A B S T R A C T
To meet various contingencies of operating wireless networks such as the change of the state of a channel, cross-layer techniques facilitate sharing of information between OSI model layers. These techniques can be apply to various protocols levels, if there are interactions for which the overall performance of the system under studies can be improved. Reliable transport protocols use the exponential back-off mechanism when a bad state of the wireless channel occurs, which temporarily blocks the transmission of data. In this paper, we suggest a new policy of timeout applied to the SCTP protocol called persistent timeout policy. This policy is based on the use of the channel status provided by the 802.11 link layer, through the cross-layer mechanism. The principle of this timeout is that when a bad state of the wireless channel blocks the sending of data, SCTP continuously observes the evolution of this state to detect the next favourable change before sending its segments. We evaluate the following two timeout policies (persistent and exponential back-off) of the SCTP protocol in an ad hoc network, and in comparison also with TCP.

Section I of this paper presents an overview of the SCTP protocol. Section II presents the principle of the persistent timeout policy. Section III presents the simulation results that are used to compare the two timeout policies of the two reliable transport protocols SCTP and TCP.
\end{abstract}

Key words: ad hoc network, SCTP retransmission, channel status.

\section{N T R O D U C T I O N}

The wireless environment is known for its operating failures as for example its dynamic random behavior, compared to the wired environment. The dynamic characteristic of ad hoc networks that are a class of wireless networks is reflected in the mobility of nodes, but especially in the unpredictable nature of the channel state that can alternate from "good" to "bad" and vice versa asynchronously. Taking into account this variation of the state of the channel in the upper layers may provide notable performance gains, such as those that have been evaluated in this proposal of persistent timeout policy.

Document [7] refers to the use of the principle of adaptation through the cross-layer design by taking into account the dynamic behavior of wireless networks. This example also deals with the flow of a multimedia traffic that is a sensitive traffic that highlights the cross-layer optimizations. The authors define three levels of adaptation according to the changes that occurs in the wireless channel: the adaptation of the transmission of application data that refers to the ability of the application to adjust its behavior when selfconfiguring may identify changes in the network and the underlying channel, the adaptation of the transmission of the segments of the transport layer protocol allowing to differentiate different types of packet loss (losses due to congestion and losses due to channel errors) for invoking congestion control and flow regulation of transmission, and then the adaptation of the network layer and link layers so that they distinguish different information flows of layers and apply the appropriate treatment depending on the required priority level. 
Retransmissions determine the reliability of the transmission of reliable transport protocols. These protocols have the particularity to address the lack of acknowledgment segments they pass by behaving in a non-persistent mechanism that consist of using the exponential back-off in the broadcasting of messages. SCTP [RFC2960] is another variant of reliable transport layer. Unlike TCP [RFC0793], SCTP has been designed from the outset to serve the same purpose as TCP in wireless environment with additional variations such as managing associations and path loss. The adaptation of TCP in wireless environment has been made with some variations, such as explicit congestion notification [3] supposed to solve the problem of using the mechanism of "congestion avoidance" in response to a poor state of wireless channel [RFC2581] [RFC2582] [RFC2883]. Despite the difference in behavior between these two transport layer protocols, a common ground is that they both use the exponential back-off mechanism for messages retransmission. From the cross-layer model in which the link layer makes available the state of the wireless channel to other layers in the environment subsystem, we propose to change the SCTP protocol through the use of persistent retransmission policy when the bad state of the channel temporarily blocks the sending of data. Persistent timeout policy applied to SCTP in a dynamic channel, yielded positive results in terms of simulation performance gain presented in Section IV below.

The proposal of the persistent timeout policy is based on the cross-layer conceptual models produced by applying the RCL method [1] [2]. The importance of that design method results in the consolidation of the achievements of the architecture. In theses documents, we have suggested to build an environment subsystem that will receive all the identified cross-layer interactions for a studied stack of protocols. In this paper, a new model of operating the SCTP protocol is proposed, that is based on the exploitation of the channel state provides by the link and physical layers.

After the presentation of variants ordained and/or reliable transport layer protocols, we devote a section of this chapter to the presentation of the operation of the SCTP protocol. The persistent timeout policy applied to the SCTP protocol is subsequently presented before the performance evaluation by simulation in ns-2 environment. A comparative study of gains between the two policies of SCTP retransmission is presented. The comparison is also made by the application of the same scenarios to the TCP protocol.

\section{OVERVIEW OF SCTP}

\section{I.1. Concepts of reliability and order}

Like TCP, SCTP provides a reliable and orderly transmission of data. UDP [RFC0768] provides neither order nor reliability. This notion of order and reliability allows distributing the family of the transport layer protocols in a two-dimensional mark where its two axes represent the criteria of order and reliability, as shown in Picture IV.1 below. This classification aims to facilitate the understanding of their functioning and especially materialize the difference between TCP and SCTP.

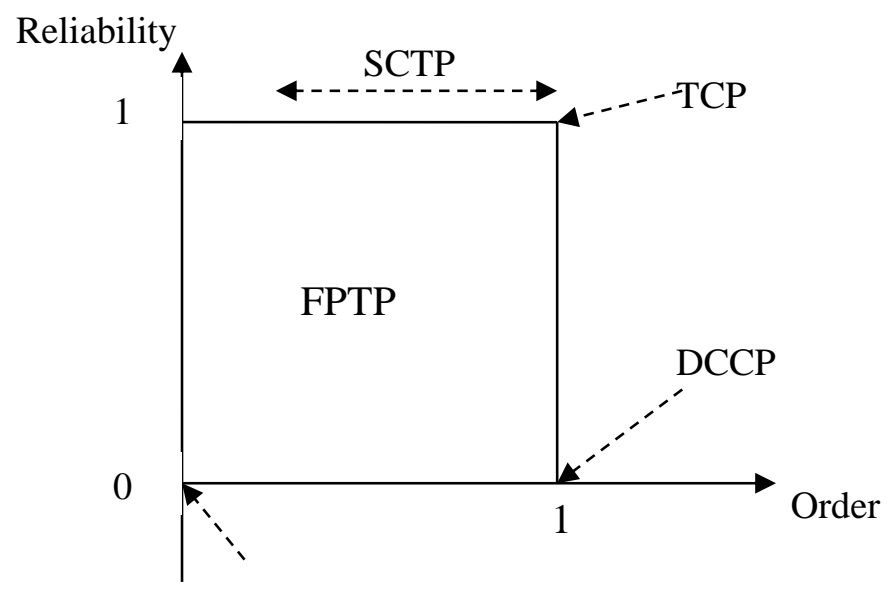




\section{UDP}

Picture I.1. The position of the transport layer protocols family

Legend :

TCP : Transmission Control Protocol

SCTP : Stream Control Transmission Protocol

FPTP : Fully Programmable Transport Protocol [8]

UDP : User Data gram Protocol

DCCP : Data gram Congestion Control Protocol [RFC4340]

SCTP uses the notion of stream and association. A stream is a sequence of messages that must be transmitted in order. An association is a broader concept than a TCP connection. It is a group in which each flow endpoint provides a list of transport addresses (@IP + port). Before transferring data, the SCTP sender and receiver execute sequences for the establishment of an association.

\section{I.2. The use of exponential back-off}

End of an association:

To end an association, the application sends the SHUTDOWN message to the SCTP. The sender then enters into the SHUTDOWN-PENDING state and remains there until the end of the transmission with acknowledgment of data waiting to be sent. After receiving the ACK of all sent data, SCTP sends a SHUTDOWN to its opposite, starts the timer T2-shutdown and then passes into the SHUTDOWN-SENT state. Upon expiry of timer sending node, another SHUTDOWN is sent, and so on until the end of the number of attempts.

At the end of data reception, the receiving node that receives a SHUTDOWN, sends a SHUTDOWN-ACK, starts its timer T2-shutdown and goes into SHUTDOWN-ACK-SENT state. Upon the expiration of the receiver's timer, another SHUTDOWN-ACK is sent, and so on until the number of attempts is reached. If the number of retries expires, the receiver removes the TCB, notifies the upper layer the inaccessibility of the opposite and entered the association in the CLOSED state.

The sender node processes the reception of the SHUTDOWN-ACK by stopping the T2-shutdown timer, by forwarding a message SHUTDOWN-COMPLETE, and by removing of the current association. When the receiving node receives the message SHUTDOWN-COMPLETE, it checks if it is in the SHUTDOWNACK-SENT state, than it stops the T2-shutdown timer, sends a SHUTDOWN-COMPLETE, and deletes the association.

The management of the two T2-shutdown timers by the sender and the receiver nodes is done by applying the traditional policy in which the retransmission timer takes increasing values multiple of the previous to limit the number of attempts. The management of the end of an association is therefore subject to optimization proposed to replace the traditional timeout by the persistent timeout policy in response of bad channel condition which results in the absence of a SHUTDOWN-ACK packet in response to a SHUTDOWN packet.

Management errors:

SCTP uses the retransmission mechanism to handle transmission errors. Retransmission data is triggered either by the expiry of the retransmission timer or by receiving a SACK indicating that the data have not been received. To reduce a potential congestion, the frequency of data retransmissions is limited. The retransmission timer RTO (Retransmission Time Out) is adjusted based on the estimation of the "round trip delay" and describes an exponential "back-off" when the loss of messages increases. The exponential 
back-off means that the timer is a multiple of the previous value for each unsuccessful attempt, as does TCP.

In an active association with a fair and consistent transmission of data, the SACK messages generate more retransmissions than the timer expiration. To reduce the possibility of unnecessary retransmissions, the rule of four SACK is used, so that the retransmission is triggered upon receipt of the fourth SACK indicating the loss of data and avoiding retransmission for cases of reordering.

\section{PERS IS TENT T IMEOUT POLICY}

\section{II.1. Principle of the persistent policy}

SCTP uses retransmission mechanism at different levels, in particular during the transmission of data and during the transmission of control messages. In each case, the expiration of the retransmission timer or the reception of a SACK indicating the lost of a packet are the main triggers of retransmissions. Retransmission triggered by the reception of a SACK is a selective retransmission of messages and is fundamentally different from the retransmission caused by the absence of SACK. The latter is triggered by the expiration of the retransmission timer. The retransmission policy used here called traditional policy is the same as that of TCP, namely the exponential back-off. Therefore, due to the use of the exponential back-off in the management of retransmissions, it becomes possible to extend the persistent timeout to the error management mechanism used by SCTP with the same TCP philosophy.

As for TCP, the exponential back-off described by the retransmission mechanism of SCTP is similar to the operation of non-persistent MAC level protocols. These protocols require a non-persistent random timeout when the channel is busy before the next attempt. In contrast to this functional philosophy, persistent protocols continue to observe the channel and transmit the frame when it is free. The state of the wireless channel is variable, which impedes the transmission of data when its status is unfavorable. Cross-layer models can make the channel state accessible to all layers through the environment subsystem. The extension of a bad channel state inevitably leads trigger retransmissions due to the expiration of the waiting ACK timer.

By analogy with the mechanism of the MAC layer for which the transmission of a frame should be done if the channel is free, the retransmission of a segment at the transport level is effective only if the state of the channel permits it. Observing the evolution of the retransmission intervals triggered by the timer that expires, the next segment retransmission attempts in the exponential back-off is closely linked to the expiry of the timer that takes a growing value multiple of its previous with each exhalation. Therefore, in this traditional policy, when the channel state temporarily blocks the transmission of data, the next attempt following the favorable change in the status of the channel will be at the expiration of the timer, which adds transmission latency. Another disadvantage of traditional politics when the bad channel state temporarily blocks the sending of data comes from a growing number of unsuccessful attempts of emission. These attempts are not negligible in terms of energy consumption. Energy determines the lifetime of the wireless network and thus assumes a crucial aspect in these networks.

To optimize the sending latency, minimize the number of unsuccessful attempts consuming energy, it is possible to apply to SCTP the principle of persistent timeout policy which, when a bad channel condition temporarily blocks the sending of data, the protocol observed continuously the variation of the state of the channel and remains open to the explicit notification of positive change in this state, instead of the principle of exponential back-off. In addition, unlike the MAC layer, the transport layer protocol applying this persistent policy is not directly subject to the risk of collision.

\section{II.2. Principle of continuing the evaluation of the channel state}


The basic principle of the persistent timeout policy depends on the continued availability of the updated status of the wireless channel provided by the link layer via the environment subsystem. In the previous cross-layer models [1][2], channel status is reflected by the parameters SNR, BER, loss rate of packets sent or received, retransmission rate, all these parameters are calculated from the activity of the physical layer. With reference to the persistent timeout, the extension of the bad state of the channel causes a silent behavior of the transport layer. This silence implies a significant reduction of the activity of the node, and this activity will be related during this period, to a probable activity of lower layers. However, the ongoing assessment of the state of the channel is necessary to allow the node to leave at the right time the silent state generated by the persistence and enable to reduce its latency. For the further assessment of the state of the channel, the node has several levels of events. There is an outdoor activity from the node that is a potential source and that the modeling must take into account. This will be done by indicating the date of updating these parameters and their recent or not judgment. On the other hand, another potential source of continuing the assessment of the channel state comes from the latent or regular activity of lower layers.

The activity related to the ambient state of high network load can meet the need of continuing the evaluation. Indeed, in such a context, there is enough activity RTS/CTS or packet transmission in the neighborhood of the node to compute the values of the state parameters of the channel, without overhead, regardless of the destination packets. Update dates calculation of these parameters will determine whether the system should enable a compensation mechanism or auxiliary diagram. This activation of the compensation mechanism is controlled by the calculation if dates are considered too old.

Contrary to the state of high load, when the network is in a light load state, the channel is most often free. Before triggering the compensation mechanism, it is necessary to take into account the mechanisms that exist within the lower layers and that ensure the continuity of the calculation of the state parameters of the channel. For example, continuity can be achieved when the used routing protocol periodically emits or when a loss of connectivity occurs (as in the case of poor channel), control messages to establish network connectivity (pro-active protocol). In this case, the auxiliary pattern is not necessary; the packets sent by the routing layer enable the physical and link layers to make a continuous assessment.

In the case of re-active routing protocols that do not emit messages for the establishment of the network topology, the compensation mechanism will consist in that the environment subsystem triggers a request to update a road that lead to particular destinations, for example, one of the locations referenced in the pending packet to be sent. The request will simply be addressed to the routing protocol. The advantage of this auxiliary scheme is that it is not necessary to define an additional protocol, but only to add a module to the environment subsystem to provide this functionality. The activity of this module will be based on the same principle of sharing network resources used by the dissemination of periodic messages. By adopting this scenario, the use of persistent timeout policy during low network load only generates a request for updating a road for one of the pending packets destination address and will thus ensure the continuity of the evaluation of the channel state.

\section{PERFORMANCE EVALUATION BY SIMULATION}

\section{III.1. Description of scenarios}

The simulated scenario is the stack of protocols composed of SCTP at transport layer, the DSR protocol and IP protocol [RFC0791] used at the network layer, IEEE 802.11 protocol for lower layers. The simulation is done in ns-2 environment [4] with a transmitting node and a receiving node. The constant traffic node issuer is subject to the variation of the channel state. The interarrival time of messages during peak is set to 0.1 second. For each TCP and SCTP, both traditional and persistent retransmission policies are evaluated with segments of 1500 bytes. 
The state model of the channel is the same. In this model, from the 200th second of the simulation, interval of availability follows a period of unavailability randomly. This randomly alternated cut model allows to match the availability of the wireless link at different times of the evolution of the traffic.

The presented simulation results are those cases in which the durations of the intervals of availability and unavailability of the link are alternately switching randomly between 20 and 100 seconds. The initial interval of 20 seconds is chosen to exceed the time of both TCP and SCTP first retransmission attempt. The evolution of these intervals applied from one scenario to another avoids intervals too close. Each scenario is evaluated 20 times, each time with a shift of the start date of the randomly alternated cut. The results are summarized by grouping durations of breaks, and by calculating the average of each evaluation for each given interval value. For example, the latency is averaged over all simulations for different intervals of interruption. Beyond the number and duration of simulations, alternated cut intervals are the main parameters to measure the influence of timeout policy being evaluated.

The objective of the simulation is to compare the performance of both TCP and SCTP, when subjected to the vagaries of a variable channel condition and to respond to changes in the state of the channel by adopting principle of persistent timeout. The simulated scenarios are identical from one protocol to another to help to make the comparison.

\section{III.2. Basis of the interpretation of the results}

We will compare the performance of both TCP and SCTP connection with the use of persistent timeout policy in response to the unavailability of the channel. The comparison criterions of the simulated scenarios are:

- We evaluate the latency of sending effective data when the channel becomes available after a period of suspension complies with the randomly alternated cut intervals.

- The criterion of theoretical maximum throughput is not shown but is related to the intuition by which improvements in latency should allow protocols to inject additional traffic.

- The criterion of unsuccessful message emissions is related to the nature of the traditional policy of retransmission based on the use of an exponential back-off. The contribution of the persistent timeout policy is measured in terms of number of retransmissions for each protocol depending on the policy chosen.

- Energy consumption is another criterion used to compare the two retransmission policies of TCP and SCTP. This consumption is considered in terms of the definition given in the work of Gallager [5]. The author defined a mobile node with a finite energy source as having a finite number of bits that it can transmit before exhausting its energy. The transmission of packets from one layer to another is an activity that also consumes energy. That is why these retransmission policies fall under the law of Gallager. We proceed to a comparison of the energy consumed by each retransmission policy.

\section{III.3. Latency curves (TCP and SCTP)}

The curves in Picture IV.2 below compare the average latency of the traditional and the persistent timeout policies of TCP and SCTP. For each protocol taken separately, the curve shows that the average latency of the traditional policy is greater than the persistent policy's. These results confirm the observations made previously in Chapter III. The crossed analysis of these results provides a comparison of the influence of variable channel state on the mechanisms of each protocol.

The first step of the crossed analysis considers the calculation of the latency in the traditional policy of both protocols. The results of the latency in traditional policy reflect the behavior of SCTP protocol that sends regular Heartbeat signaling packets. These regular shipments allow protocol to quickly detect the change of the channel state without waiting for the expiry of the timer as does TCP. Rapid detection of this change 
is a benefit of the use of sending periodic Heartbeat packets. However, the protocol is disabled by setting the availability of the destination node by exchanging these signaling messages before transferring the effective data. This phase of certification of the availability of the destination node extends the SCTP latency. The basis of the comparison is made from the sending of effective data. Despite differences, the curves of latency for the traditional policy give mixed results in favor of one or the other of the two protocols, depending on the time of the channel availability, on the expiration of the retransmission timer of TCP and at end, of the Heartbeat packet exchange of SCTP protocol.

The second step of the crossed analysis refers to the calculation of the latency of the persistent timeout policy of the two protocols. The transmission scheme of the data at every expiration of the timer is replaced by a persistent behavior. Both protocols observe the next favorable change in the channel state before sending data. The management mechanism of the failure of the path is also modified to take in account the variable state of the channel. This mechanism is disabled when the channel is in a bad state and is reactivated as soon as the state of the channel becomes favorable again, at the same time as the mechanism for sending data. Therefore, during the persistence, the sending of Heartbeat messages is also suspended. The observed behavior is that the protocol uses the exchange of Heartbeat packets before sending data to certify the availability of the destination node. This is why the average latency in the persistent timeout policy is improved by TCP that sends its data as soon as the channel condition becomes favorable while SCTP is hampered by the exchange of Heartbeat messages before sending data messages.

The use of the Heartbeat message allows testing the availability of a destination. In our case, the two nodes are in sight. Therefore, the good state of the channel is enough to ensure the transmission of information. This is why in this case, the delay of TCP with persistent timeout is better than SCTP that is slowed by Heartbeat messages. Conversely, in the case of traditional policy, the Heartbeat messages find all their interest and lead to better outcomes for SCTP.
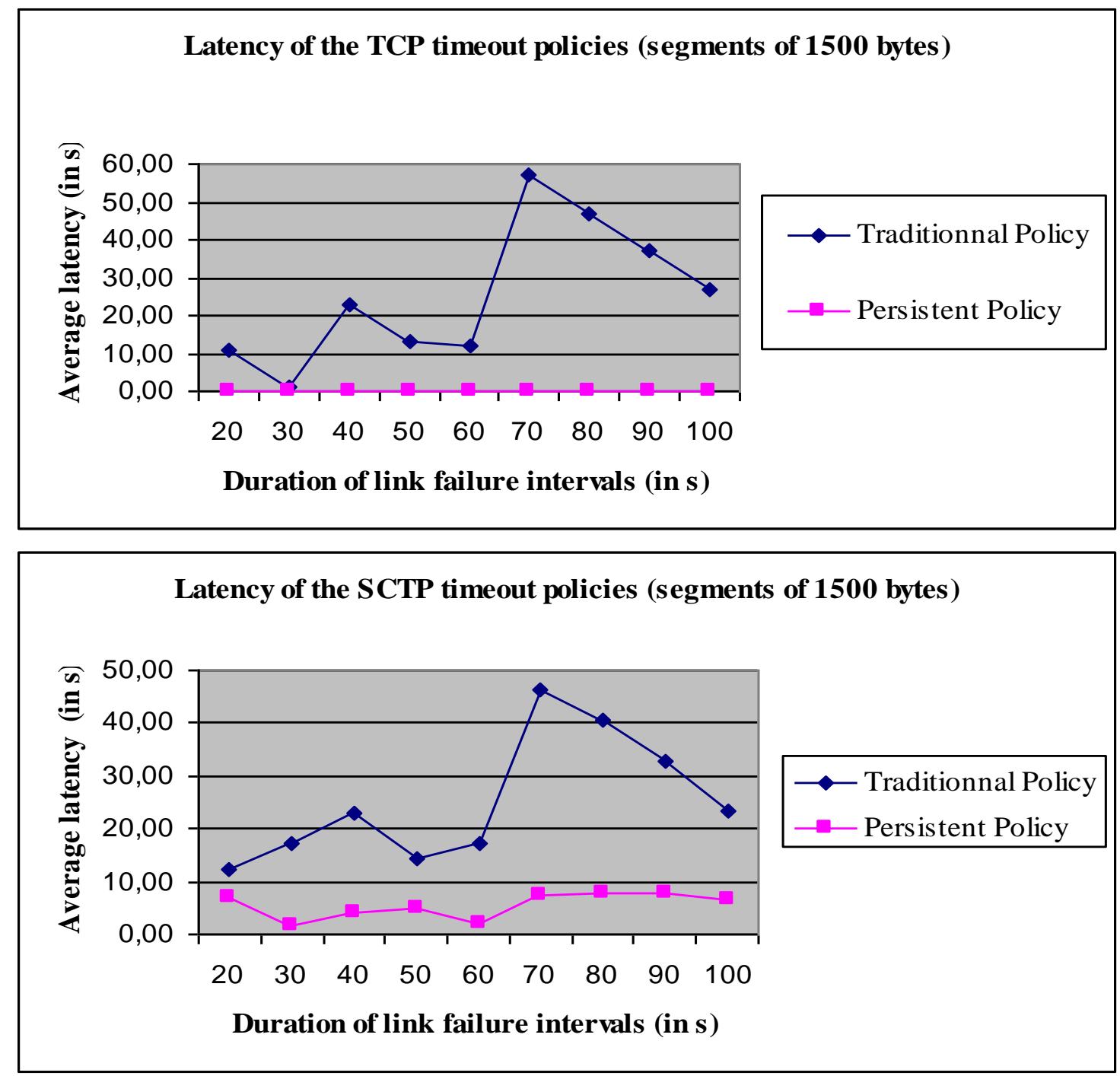
Picture III.2. Compared evolution of the average latency of TCP and SCTP timeout policies

\section{I .4. Energy consumed by unsuccessul emissions}

The exponential back-off results in transmission attempts at every expiration of the sending timer. To limit the number of attempts in the traditional pattern, the principle is used to evolve exponentially the waiting time between two consecutive retransmission attempts. In the case of unavailability due to poor channel condition, the traditional policy of retransmission used generates retransmissions of messages that were unsuccessful. However, these programs have a notorious influence on energy consumption, since mobile nodes have a finite energy source. TCP retransmits data segments for each attempt while SCTP adds to the emission of these unsuccessful data messages, the transmission equally unsuccessful of the signaling Heartbeat messages.

SCTP retransmission policy extends the traditional as described for the data segments in the case of a multihomed node. In addition to this policy in which SCTP retransmits the data message to a replacement address on the expiry of the sending timer, the Heartbeat message is sent immediately to the destination that generates the expiration. In normal times, the Heartbeat additional messages provide a mechanism for the sender to update more frequently the estimated RTT of the alternative destination, which gives a better RTT based on which the RTO is calculated.

The traditional timing process of SCTP is different from TCP. For example, when a message is lost during transmission to its original purpose, it is forwarded to the destination later parts. If the retransmission timer expires for this last destination, the lost message is retransmitted again to another alternative destination if it exists, otherwise to the primary destination. In addition to these multiple shipments data messages to these addresses, Heartbeat message is also sent to the destination that has generated the timer expiration. By this mechanism, SCTP causes more retransmission attempts than TCP when the bad state of the channel extends.

The results observed on the curves of Picture IV.3 shows for TCP and SCTP taken in isolation, as expected, the advantage of persistent retransmission policy on traditional policy in terms of reducing the energy consumption by the number of retransmission attempts. The crossed analysis of the results will assess the behavior of each protocol. Due to the difference in operation previously stated, the expected benefit in the sense of reducing the number of unsuccessful attempts of any type of packet is issued in favor of TCP at the expense of SCTP for both retransmission policies. Picture IV.3 dedicated study on data packets only, which seems to give an advantage to traditional SCTP policy because it emits less data packets by using the detection of the path failure. And one of the drawbacks of this mechanism of path failure detection is that SCTP Heartbeat messages are issued regularly even when the channel is not available because this information from the link layer is unknown to the mechanism. As previously stated, the unavailability of the channel that generates the lack of acknowledgment requires the use of SCTP retransmissions of data messages (which proved unsuccessful) at each end of the retransmission timer.

To reduce the imbalance to the detriment of the SCTP protocol due to the transmission of Heartbeat messages at every expiration of the timer, the comparison between the two protocols for each retransmission policy set is made on the basis of emissions of data messages on the curves of FIG IV.3. Because SCTP protocol is disabled by issuing Heartbeat messages, it sends less data messages than TCP in traditional policy. Continuing the comparison of the two protocols with the persistent policy, the number 
of unsuccessful emissions remains the same, since no data packet is sent by either of the two protocols for the unavailability of the channel, not even the SCTP Heartbeat messages in this option of the simulation. Addressing unsuccessful emissions regardless of the message type shows greater energy consumption in SCTP persistent and traditional timeout policies.
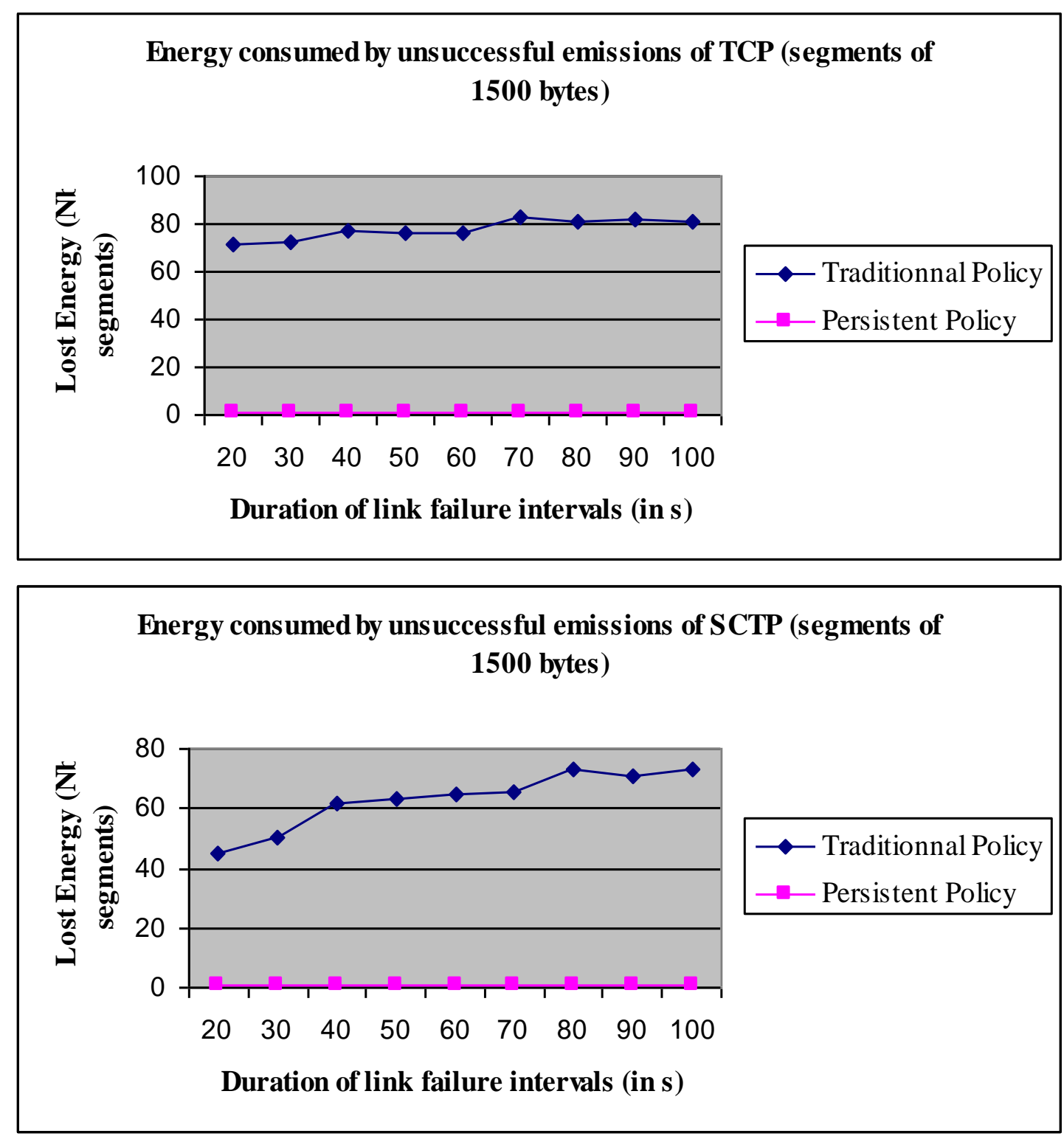
Picture III.3. Compared evolution of the unsuccessful emissions attempts of TCP and SCTP protocols according to the timeout policies.

\section{III.5. Percentage of unsuccessul emissions compared to the data transferred}

Unsuccessful emissions are part of the retransmission mechanism used by both TCP and SCTP to ensure reliable data transmission. These emissions are generated by the unsuccessful extension of the bad state of the channel, embodied here by the randomly alternated cut model. The curves in Picture IV.3 above demonstrate that TCP performs more unsuccessful emissions of data packets compared to SCTP subject to the emission mechanism of signaling messages. It is therefore interesting to evaluate the ratio between the number of attempts and the total number of transferred data to better assess the effectiveness of each protocol. Unsuccessful attempts, although energy consumers are justified to ensure reliable transfer of the data set. It may be interesting, in terms of comparing the two protocols, striking a balance between the number of attempts and the amount of transferred data, particularly in the case of traditional policy, since persistent policy increases the number of sent segments with fewer attempts and less energy consumption.

The crossed analysis of the simulation results given by the curves in Picture IV.4 below allows assessing the performance of each protocol when the number of attempts is reduced to the percentage of 
data messages received at the destination. Whereas calculating the rate of the unsuccessful emissions that consumes energy depending on the amount of transferred data, TCP is much favored by higher rate than the SCTP, which has a lower bit rate. For example, in the case of traditional timeout for the alternating intervals of 100 seconds of unavailability of the channel, the TCP generates less than $0.6 \%$ of unsuccessful emissions compared to the volume of transferred data and the protocol SCTP is about $1.25 \%$. The curves of the persistent timeout policy also shows the advantage for TCP despite the use of SCTP results without the great part of the unsuccessful emissions devoted to the issue of signaling messages.
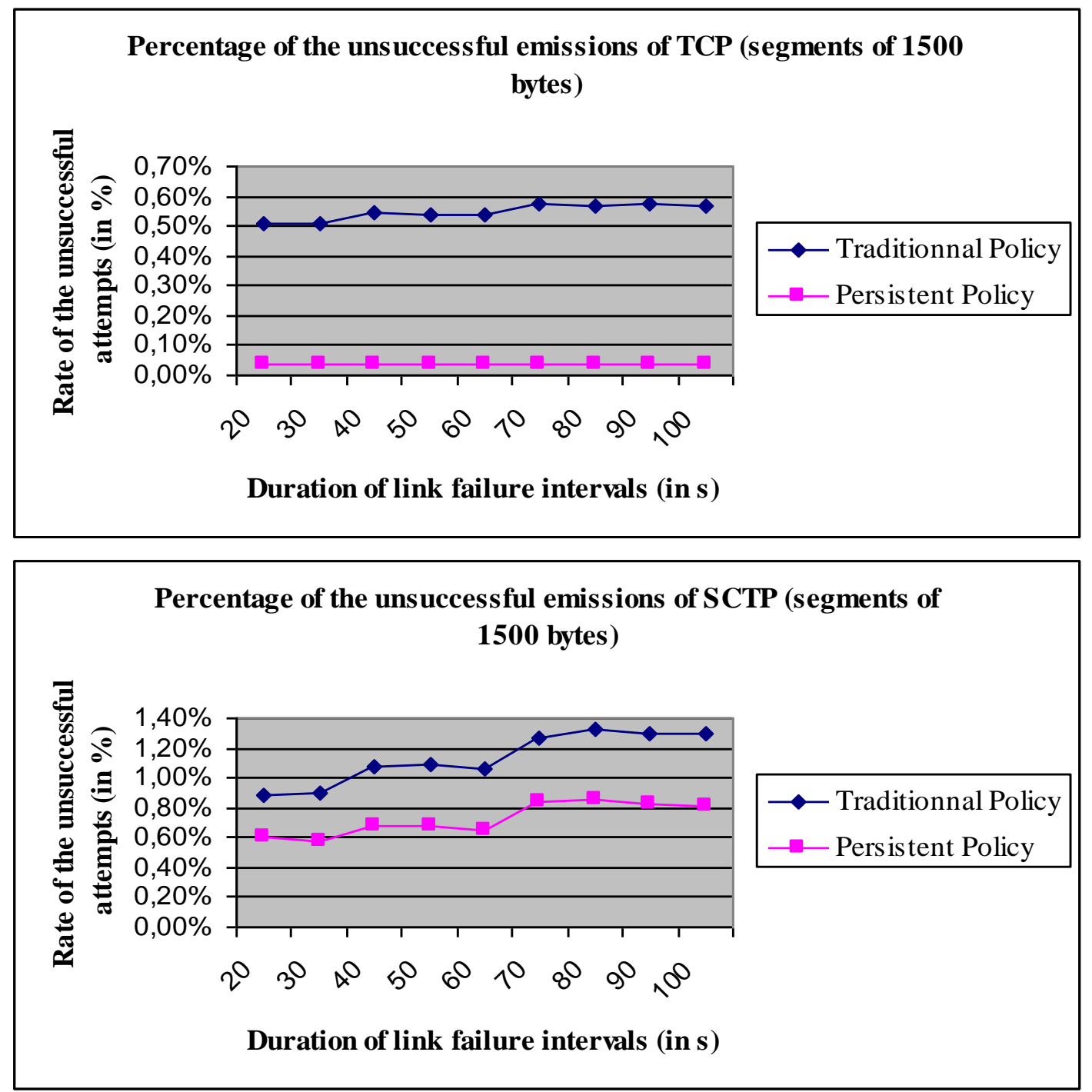

Picture III.4. Percentage of the unsuccessful emissions by the TCP and SCTP transferred data volume 


\section{O N CLUS I O N}

The management of association mechanism of the SCTP protocol which is associated with the path loss detection mechanism in the network, by sending periodic messages, are just some few points of difference in behavior which exist with TCP. The persistent timeout policy applied to TCP and SCTP protocols adequately replaces the exponential back-off mechanism described by the retransmission of the two protocols, when the channel state is bad. It is based on the channel status information provided by the link layer via the parameters of the environment subsystem and the explicit notification modules it contains. The results showed the advantage of the persistent timeout policy when the mobile node is subject to the vagaries of the dynamic variation of the channel state. This advantage is reflected in terms of latency, throughput and energy consumption due to retransmission attempts. Cross-comparison of results for each protocol reflects the effectiveness of both TCP and SCTP which constitute their main points of difference. Additional work to be carried out is for studying the behavior of other protocols in the stack to propose cross-layer models that avoid the duplication of mechanisms from one layer to another. This study will also establish the mechanism for a continuous assessment of the channel state on which is based the persistent timeout policy. To enable the system to provide updated information about the status of the channel and thus serve the persistent timeout policy, the overall performance should be studied and necessary complementary mechanisms proposed. 


\section{R E F E R E N C E S}

[1] M. I. Tiado, R. Dhaou, A-L. Beylot. "Multilevel Network Modelling to Achieve Cross-layer Mechanisms", Med-Hoc-Net'05 Conference, Île de Porquerolles, France, IFIP Serie, pp79-89, Springer 2006.

[2] M. Issoufou Tiado, R. Dhaou, A.-L. Beylot, "RCL: A new Method for Cross-Layer Network Modelling and Simulation", In: 7th IFIP International Conference on Mobile and Wireless Communication Networks, MWCN'05, Marrakech, Maroc, Septembre 2005, to appear.

[3] K. Ramakrishnan, S. Floyd, D. Black. "The Addition of Explicit Congestion Notification (ECN) to IP” RFC3168 September 2001, www.ietf.org

[4] K. Fall and K. Varadhan. "The ns manual, the VINT Project" http://www.isi.edu.nsnam/ns/doc/index.html, December 2003.

[5] R. G. Gallager, "Energy limited channels : coding, Multi - access, and Spread Spectrum", 1998 Conf. Info. Sci Sys. Mar 1998

[6] V. Kawadia, P.R. Kumar, "A cautionary perspective on cross-layer design", IEEE Wireless Communications, Volume 12, Issue 1, Feb. 2005, Page(s):3 - 11.

[7] H. Zheng, "Optimising Wireless Multimedia Transmissions Through Cross-layer Design", Multimedia and Expo, 2003. ICME '03. Proceedings. Volume 1, 6-9 July 2003 Page(s):I - 185 -8 vol.1.

[8] P. Senac, M. Diaz, "FPTP : the XQoS aware and fully programmable trasnport protocol", ICON2003 Networks, 28 sept - 1 oct 2003, the 11h IEEE International Conference, p249-254

[RFC2581] M. Allman, V. Paxon, W. Stevens, “TCP Congestion Control”, RFC 2581, April 1999.

[RFC2582] S. Floyd, T. Henderson, "The NewReno Modification to TCP's Fast Recovery Algorithm", RFC 2582, April 1999.

[RFC2883] S. Floyd, J. Mahdavi, M. Mathis, M. Podlosky, "An Extension to the Selective Acknowledgement (SACK) Option for TCP”, RFC 2883, August 1999.

[RFC2960] R. Stewart, Q. Xie, K. Morneault, C. Sharp, H. Schwarzbauer, T. Taylor, I. Rytina, M. Kalla, L. Zhang, V. Paxson, "Stream Control Transmission Protocol", RFC 2960, October 2000.

[RFC0768] J. Postel , "User Datagram Protocol", STD 6, RFC 768, August 1980.

[RFC0791] J. Postel. “Internet Protocol”, RFC 0791, Sep-01-1981, www.ietf.org.

[RFC0793] J. Postel. “Transmission Control Protocol”, RFC 0793 Sep-01-1981, www.ietf.org.

[RFC4340] E. Kolher, M. Handley, S. Floyd, "Datagram Congestion Control Protocol (DCCP)", RFC4340, March 2006, http://tools.ietf.org/html/rfc4340 